\title{
Slow light propagation in trapped atomic quantum gases
}

\author{
Özgür E. Müstecaplığlu and L. You \\ School of Physics, Georgia Institute of Technology, Atlanta GA 30309-0432, USA
}

(November 3, 2018)

\begin{abstract}
We study semi-classical slow light propagation in trapped two level atomic quantum gases. The temperature dependent behaviors of both group velocity and transmissions are compared for low temperature Bose, Fermi, and Boltzman gases within the local density approximation for their spatial density profile.

03.75.Fi,42.65.An,42.50.Gy
\end{abstract}

Recently, a dramatic demonstration of slow light propagation, down to $17(\mathrm{~m} / \mathrm{s})$ was reported [1,2]. Achievement of such an extremely low speed owes mostly to the widely discussed phenomenon of electromagnetically induced transparency (EIT), which makes propagation of light in an otherwise opaque medium possible [3]. In related theoretical and experimental studies, three level atomic vapours are the typical medium. Although BoseCondensation is not crucial to EIT or slow light propagation [1]:2], the long coherence time of such a degenerate quantum medium does prove to be advantageous. Similar observations have since been carried out in optically dense hot rubidium vapours $[\sim 90(\mathrm{~m} / \mathrm{s})]$ 任 and in a optically thick Pr:YSO crystal $[\sim 45(\mathrm{~m} / \mathrm{s})]$ [5]. Possibilities of vanishing or even negative group velocities, in a coherently driven Doppler broadened atomic vapours have also been discussed [f].

Ultra-slow light propagation offers many potential applications since macroscopically the medium can be viewed as possessing a high index of refraction, albeit within a narrow spectrum. The accompanied superhigh nonlinear coupling between weak fields in a long coherence medium such as a Bose-Einstein condensate (BEC) opens up novel regimes of quantum nonlinear optics [7]. Application to quantum networks and quantum information processing, including quantum entanglement of slow photons [8], non-classical (e.g. squeezed) and entangled atomic ensembles [9], quantum memories [10], have been proposed. Their implications to quantum non-demolishing measurements and high precision spectroscopy using squeezed light have been suggested through enhanced acousto-optical effects 11] and narrow-band sources for non-classical radiation [12].

The aim of this paper is two fold: (1) Inspired by the recent theoretical modeling of slow light propagation [1] in BEC by Morigi and Agarwal [13], we ask the question of comparative differences related to spatial density profiles of different quantum gases; (2) We explore a simpler model composed of two level atoms 114. In our formulation, we consider the semi-classical propagation of a laser pulse through an ultra-cold quantum gas of two level atoms described by a density profile $\rho(\vec{r}, t)$. At very cold temperatures atoms are highly delocalized and continuum treatment of the gas as a medium is desirable. The polarization density operator is thus 
$\vec{P}(\vec{r}, t)=\vec{d}_{g e} \psi_{g}^{\dagger}(\vec{r}, t) \psi_{e}(\vec{r}, t)+$ h.c. [15] with $\vec{d}_{g e}$ the electronic dipole transition moment between ground state $|g\rangle$ and excited state $|e\rangle \cdot \psi_{\eta=g, e}(\vec{r}, t)$ is the second quantized field operator for atoms in state $|g\rangle$ and $|e\rangle$ respectively. An incident laser pulse polarizes the medium and the induced dipoles of atoms subsequently emit coherent radiation. For most of aforementioned applications, the atomic density profile remains an essential ingredient. In addition, careful determination of the optical response inside a dense medium requires the inclusion of local field corrections. In case of two level atoms as studied here, it is necessary to also have a significant transmission through the medium. At any point within the medium, the electric field is given by the superposition of the incident field and the radiated field, the latter excludes the self-field radiated by the dipole at this same point. Adopting the standard approach, we write the total local field acting on the atoms $\vec{E}_{L}=\vec{E}_{P}+\vec{E}_{D}+\vec{E}_{V}$, as a sum of the incident field $\left(\vec{E}_{P}\right)$, the field generated by other atomic dipoles $\left(\vec{E}_{D}\right)$ and the field due to vacuum fluctuations $\left(\vec{E}_{V}\right)$. The dipole field depends on the type of medium as well as its density profile.

Following standard procedures [16] we eliminate fast radiation field dynamics. It is then possible to recognize that $\vec{E}_{D}$ is given by $\left[\vec{P}_{t}\right]$, the retarded transverse polarization field [15, 17]. We obtain

$$
\vec{E}_{D}(\vec{r}, t)=\vec{\nabla} \times \vec{\nabla} \times \int_{V /\{\vec{r}\}} d \vec{r}^{\prime} \frac{\vec{P}\left(\vec{r}^{\prime}, t-\left|\vec{r}-\vec{r}^{\prime}\right| / c\right)}{\left|\vec{r}-\vec{r}^{\prime}\right|},
$$

where $V /\{\vec{r}\}$ indicates that integral over the interaction region excludes a small region around $\vec{r}$. Proper evaluation of the integral in the vicinity of $\vec{r}$ yields the LorentzLorenz correction (local field correction) [17],

$$
\vec{E}_{L}=\vec{E}+\frac{4 \pi}{3} \vec{P}
$$

The field $\vec{E}$, including contributions of all dipoles in the interaction volume $V$, is the macroscopic field governed by the Maxwell equations. It is important to point out that the Lorentz-Lorenz relation Eq. (11) holds true for both linear and nonlinear media [18]. To maintain sufficient transmission, we assume the incident field (of central frequency $\omega$ ) to be far-off resonant (from atomic resonance $\omega_{0}$ ) with a large detuning $\Delta=\omega-\omega_{0}$. When the incident pulse is weak, the linear response theory probes the unperturbed density profile $\rho(\vec{r}, t) \approx \rho(\vec{r})=$ $\psi_{g}^{\dagger}(\vec{r}, 0) \psi_{g}(\vec{r}, 0)$. For stronger higher input fields, the density of the gas needs to be determined self-consistently [19]. The linear optical response function (the electric susceptibility, $\chi$ ) follows the Clausius-Mossotti relation

$$
\chi(\vec{r})=\frac{\alpha \rho(\vec{r})}{1-\frac{4 \pi}{3} \alpha \rho(\vec{r})+i \frac{\gamma}{2 \Delta}},
$$

involving Lorentz-Lorenz shift as a first correction [15, 19. 24. $\alpha=\left|\vec{d}_{g e}\right|^{2} / \hbar \Delta$ is the approximate offresonant atomic polarizability and $\gamma$ is the excited state 
spontaneous emission rate. Higher order quantum corrections to this formula arise from multiple scattering of photons within neighboring atomic pairs as well as from many-body correlations. These effects are neglected in this study for the coherent propagation effect. Recent studies of these corrections found the weak field optical response Eq. (2) works well in available experimental density regimes for both Bose [15,19,24 and Fermi gases 24,25. The main physical reason allowing for such a simplification is the experimental effort in assuring a low optical density $\chi(\vec{r})=\alpha \rho(\vec{r})$ to provide reliable probes of the system. Local field correction is significant when the number of atoms in a characteristic volume

$$
V_{\alpha}=\frac{4 \pi}{3} \alpha=4 \pi^{2} \frac{\gamma}{\Delta k_{L}^{3}},
$$

is comparable to unity. Here, $k_{L}$ is the wave number of the near-resonant incident field with detuning a $\Delta(\approx 10 \gamma$ in this study) from the transition wavelength $\lambda=589$ (nm). This gives $V_{\alpha} \approx 4 \times 10^{-15} \mathrm{~cm}^{3}$. Hence, only for densities around $10^{14} \mathrm{~cm}^{-3}$ or higher, will local field start to cause qualitative/quantitative modifications to the optical response of an atomic cloud. For a non-interacting condensate of $10^{6}$ atoms, it is estimated [13] using trap parameters of Ref. [1] that an equivalent homogeneous density is $\rho \approx 8 \times 10^{15} \mathrm{~cm}^{-3}$. The presence of a repulsive interaction between atoms lowers that value to $\rho \approx 3 \times 10^{13} \mathrm{~cm}^{-3}$ (using Thomas-Fermi approximation). Therefore, it is more serious to neglect local field effects in a BEC model of non-interacting atoms than our semiclassical treatment for an interacting condensate. Although the above estimates could justify the neglect of Lorentz-Lorenz shift, we decided to keep it for a couple of reasons. First, these estimates rely on a homogeneous density profile. Yet, the density of an actual condensate can be much higher near the trap center. For an inhomogeneous condensate the group velocity is determined by a spatial averaged optical response, it is therefore necessary to check consistently any deviations from the column density approach used for a homogeneous density distribution. Second, considerable arguments (see discussions in next paragraph) point to the fact that local field effects, many body correlations, and multiple scattering effects are of the same order. Keeping Lorentz-Lorenz shift thus allows for a consistent check against approximations in neglecting the many body (e.g. second order density-density) correlations as well as multiple scattering.

It is a challenging task to include quantum many-body correlations and multiple scattering of the medium into an effective optical response (effective refractive index). In an earlier attempt, a perturbative density expansion, similar to the Virial expansion, was employed in Ref. [20] to calculate the first two corrections. The effect was found to be negligible for atomic densities $\ll k_{L}^{3}$. Later, it was shown that such an approximation is equivalent to truncating an infinite hierarchy of equations for correlation functions [21,22,25]. Furthermore, it was shown 
under the truncation approximation that correlation correction terms are of the same order as the local field corrections. A notable exception is the low density Fermi gas in a Cooper paired BCS state [26], a case not considered here. The neglect of quantum correlations even in the ideal fermi gas case as studied here may affect some of our results. Other recent studies include self-consistent analysis in [15, 19,24 and a Dyson equation formulation in 23.

The real part of the susceptibility $\left(\chi^{\prime}\right)$ is related to the index of refraction from which we can calculate the group velocity according to [4]

$$
v_{g}(\omega, \vec{r}) \approx \frac{c}{1+2 \pi \chi^{\prime}+2 \pi \omega \frac{\partial \chi^{\prime}}{\partial \omega}}
$$

where $c$ is the speed of light in vacuum, and the probe pulse central wave number is $k=\omega / c$. In obtaining the above equation, we have neglected a small contribution from $\partial \chi^{\prime} / \partial k$, and assumed the susceptibility changes slowly over an optical wavelength $2 \pi / k$. The result is,

$$
v_{g}=c\left(1+\frac{2 \pi \omega_{0} \alpha \rho}{\Delta(1-4 \pi \alpha \rho / 3)^{2}}\right)^{-1} .
$$

In the reported measurements of slow group velocity [1,2], two separate parameters are needed: 1) the effective propagation length of the medium; 2) the delay time of the probe pulse. The ratio of these two quantities defines the effective group velocity [1]. We adopted the definitions as used by Morigi and Agwaral [13] for a three level Bose gas, and appropriately generalized to the case of an interacting Bose gas. We then compare with corresponding results for an (ideal) Fermi and Boltzman gas.

The ground state atomic density profile for a Fermi gas is obtained following the semi-classical approximation given by

$$
\rho_{F}(\vec{r})=\int \frac{d \vec{p}}{h^{3}} \frac{1}{e^{\beta[H(\vec{p}, \vec{r})-\mu]}+1},
$$

where $\beta=1 /\left(k_{B} T\right)$. The Hamiltonian $H(\vec{p}, \vec{r})$ describes ground state atomic motion inside an external trapping potential $V(\vec{r})=M \omega_{r}^{2}\left(r^{2}+\epsilon^{2} z^{2}\right) / 2 . \omega_{r}$ is the radial trap frequency, $\epsilon$ is the aspect ratio, and $M$ is the atomic mass. The probe light is assumed to propagate along the (long) $z$-axis of the atomic cloud. The chemical potential $\mu$ is determined by the normalization $N=\int d \vec{r} \rho_{F}(\vec{r})$ with $N$ the total number of atoms. In the low temperature limit, $\mu$ can be described within the Sommerfeld expansion, while the high temperature behavior is found by direct integration similar to a Boltzmann gas [27,

$$
\mu= \begin{cases}-\ln \left[6\left(T / T_{F}\right)^{3}\right] / \beta, & T>0.55 T_{F}, \\ E_{F}\left[1-\pi^{2}\left(T / T_{F}\right)^{2} / 3\right], & T \leq 0.55 T_{F},\end{cases}
$$

where $T_{F}$ and $E_{F}$ are the Fermi temperature and Fermi energy.

For an interacting Bose gas, the ground state density profile is computed using the analytic fitting function 
as developed in [28]. Below the condensation temperature $T_{C}$, the condensate component is described by the Thomas-Fermi approximation [28,29],

$$
\rho_{B}(\vec{r})=\frac{\mu-V}{U} \theta(\mu-V) \theta\left(T_{c}-T\right)+\frac{g_{3 / 2}\left(z e^{-\beta V}\right)}{\Lambda_{T}^{3}},
$$

with $T_{c}$ is the critical temperature. $U=4 \pi \hbar^{2} a_{\mathrm{sc}} / M$ with $a_{\mathrm{sc}}$ the atomic scattering length. $\theta($.$) is the Heaviside step$ function, $g_{n}(x)=\sum_{j} x^{j} / j^{n}$, and $\Lambda_{T}$ is the thermal de Bröglie wavelength. The chemical potential at high temperatures is determined by solving $\mathrm{Li}_{3}(\mathbf{z})=\left(T / T_{c}\right)^{-3} \zeta(3)$ in terms of the fugacity $z=e^{\beta \mu} . \operatorname{Li}_{3}($.$) and \zeta(3)$ are the third order polylogarithmic and Riemann-Zeta functions respectively. At low temperatures, it is found that 28,

$$
\mu=\mu_{T F}\left(\frac{N_{0}}{N}\right)^{2 / 5}
$$

with $\mu_{T F}$ the Thomas-Fermi approximation chemical potential. The condensate fraction is then given by

$$
\frac{N_{0}}{N}=1-\left(\frac{T}{T_{c}}\right)^{3}-\eta \frac{\zeta(2)}{\zeta(3)}\left(\frac{T}{T_{c}}\right)^{2}\left[1-\left(\frac{T}{T_{c}}\right)^{3}\right]^{2 / 5}
$$

with a scaling parameter $\eta$ defined as

$$
\eta=\frac{\mu_{T F}}{k_{B} T_{c}}=\frac{1}{2} \zeta(3)^{1 / 3}\left(15 N^{1 / 6} \frac{a_{\mathrm{sc}}}{a_{h o}}\right)^{2 / 5} .
$$

$a_{\mathrm{ho}}=\sqrt{\hbar /\left(M \epsilon^{1 / 3} \omega_{r}\right)}$ is the average harmonic oscillator length scale [30].

Finally, for a classical gas we use

$$
\rho_{C}(\vec{r})=\frac{1}{\Lambda_{T}^{3}} e^{-\beta(V-\mu)},
$$

with the chemical potential $\mu=-\ln \left[6\left(T / T_{F}\right)^{3}\right] / \beta$.

From each of the above three distributions for $\rho(\vec{r})$, we first determine the effective length of the medium using

$$
L(T)=\left[\int_{V} d \vec{r} z^{2} \rho(\vec{r})\right]^{1 / 2} .
$$

In a typical experiment, a pinhole (of radius $R$ less than the Thomas Fermi radius) is often introduced just after the atomic cloud to restrict delay time detection to only light passing through the most dense central axial column. This can be included by the averaged delay time

$$
t_{d}(T)=\frac{1}{\pi R^{2}} \int_{0}^{R} 2 \pi d r \int_{-L}^{L} d z \frac{1}{v_{g}(\vec{r})}-\frac{2 L}{c} .
$$

For small pinhole size and long optical path length $L$, we can ignore the spatial dependence of $L$ due to shape of the cloud [13]. The group speed is then calculated according to $v_{g}=L(T) / t_{d}(T)$. Typical reports of our 
calculation are reported in Fig. 1. Below the BEC transition temperature we see that the group velocity in a Bose gas drops sharply. Deviations of Fermi gas from the classical behavior, on the other hand, appear below

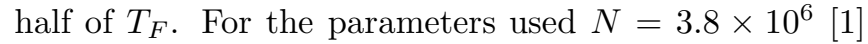
and other parameters as given in Fig. 1 caption, $T_{F}$ 31] is approximately twice the $T_{c}$. In such degenerate low temperature regimes, different quantum statistics of the gases result in qualitatively and quantitatively different behaviors of their slowed group velocities. For this set of experimental parameters, we found local field correction causes only $\sim 3 \%$ reduction of group velocity, invisible in the figure.

The absorption caused by the imaginary part of the susceptibility $\left(\chi^{\prime \prime}\right)$ is best described by the the transmission coefficient

$$
\alpha_{\mathcal{T}}=-2 \frac{\omega_{0}}{c} \frac{1}{\pi R^{2}} \int_{0}^{R} 2 \pi r d r \int_{-L(T) / 2}^{L(T) / 2} d z \chi^{\prime \prime},
$$

which in turn gives the transmission $\mathcal{T}(\Delta, T)=e^{\alpha \mathcal{T}}$. A good estimate is provided by $\mathcal{T}=e^{-2 \omega_{0} \chi_{m}^{\prime \prime} L(T)}$, with $\chi_{m}^{\prime \prime}$ the imaginary part at the peak density (at $\vec{r}=0)$. Our numerical results are presented in Fig. 2 for $T \sim T_{c} / 2 \approx$ $T_{F} / 4$, in the quantum degenerate regime. We notice the transmission for a Bose gas is always lowest, while for the corresponding Fermi is always the highest. This can be simply explained in terms if their peak densities. For near-resonant pulse propagation with $|\Delta|<3 \gamma$ Bose gas becomes essentially opaque, while significant transmission through a Fermi gas is still possible. This high transmission feature can be considered as an advantage of a Fermi gas, over the Bose gas regarding applications in quantum memories, and optical data storage.

It is interesting to discuss briefly results for the zero temperature limit, in which analytical solutions exist for all density distribution functions, given respectively by

$$
\begin{aligned}
& \rho_{F}(T=0)=\frac{8 N \epsilon}{\pi^{2} R_{F}^{6}}\left(R_{F}^{2}-r^{2}-\epsilon^{2} z^{2}\right)^{3 / 2}, \\
& \rho_{B}(T=0)=\frac{15 N \epsilon}{8 \pi R_{B}^{5}}\left(R_{B}^{2}-r^{2}-\epsilon^{2} z^{2}\right),
\end{aligned}
$$

where $R_{B}=\left(15 N \epsilon a_{\mathrm{sc}} / a_{\mathrm{ho}}\right)^{1 / 5} a_{r}$ and $R_{F}=(48 N \epsilon)^{1 / 6} a_{r}$ are the Thomas-Fermi radius for bosonic and fermionic clouds and $a_{r}=\sqrt{\hbar /\left(M \omega_{r}\right)}$.

After some tedious calculations, we obtain

$$
v_{g}=\frac{4 \omega_{0}^{2} \Delta^{2}}{3 \sqrt{7} N \epsilon c^{2} \gamma} R^{2} R_{B}\left(1-\left[1-\left(\frac{R}{R_{B}}\right)^{2}\right]^{5 / 2}\right)^{-1}
$$

for bose gas and

$$
v_{g}=\frac{\sqrt{2} \omega_{0}^{2} \Delta^{2}}{9 N \epsilon c^{2} \gamma} R_{F}^{3}\left[1-\left(\frac{R}{R_{F}}\right)^{2}+\frac{1}{3}\left(\frac{R}{R_{F}}\right)^{4}\right]^{-1}
$$

for fermi gas respectively at $T=0$.. 
Valuable insight can be gained by examining the external parameter dependence of the above two formulae. Most notably $v_{g} \sim N^{-2 / 5}$ for a Bose gas while $v_{g} \sim N^{-1 / 2}$ for a Fermi gas. Larger delay times are obtained for $R \ll R_{F}, R_{B}$ than for $R \sim R_{F}, R_{B}$, and depends on the statistical nature of the atomic medium. In a Fermi gas, $v_{g}$ is three times larger for $R \sim R_{F}$ than for $R \ll R_{F}$. While in the case of Bose gas, $v_{g}$ is 2.5 times larger for $R \sim R_{B}$ than for $R \ll R_{B}$. This dependence may be used to calibrate and determine experimental observables such as the cloud size, temperature, and the Thomas-Fermi radius.

In conclusion, we have examined the temperature dependent behavior of slow light transmission through an ultra-cold quantum gas of two level atoms. We have included local field corrections, and compared the results for atoms with different quantum statistics. Analytical results are obtained below the condensation and Fermi temperatures. It is interesting to point out that as displayed in Fig. 1, the logarithmic of group velocity for a Fermi gas has a simple temperature dependence; it is almost linear. This feature may be used for precise temperature measurements in regimes below the Fermi temperature when precise calorimetry is difficult to achieve. The absolute scale can be obtained by a calibration against other independent measurements at higher temperatures. Furthermore, as emphasized in the theoretical studies in Ref. [13], a sharp discontinuity shows up for a Bose gas exactly at the condensation phase transition point. This is clearly displayed in Fig. 11. For a three-level system, theoretical results for $T<T_{C}$ are less reliable because of complications from both strong mean-field mutual couplings and multiple scattering effects. Consequently, agreement with experiment in this regime is poor 133. In the two-level case studied here, our theory includes the self-mean-field interaction, and one may expect its validity even in this ultra-low temperature regime as long as the incident probe field is weak. Unfortunately, there is no current experimental data to compare with. Should this prove to be true, this dramatic feature at the transition temperature can be used for accurate determination of $T_{C}$. Finally we note that although these results are similar to those in three level EIT systems. The two level model studied here may present some practical experimental advantages.

We acknowledge insightful discussions with Dr. P. Zhou. This work is supported by the NSF grant No. PHY-9722410..

[1] L. V. Hau, S. E. Harris, Z. Dutton, and C. H. Behroozi, Nature 397, 594 (1999). See also Ref. [2] where an average group velocity as low as $1(\mathrm{~m} / \mathrm{s})$ was reported.

[2] S. Inouye, R. F. Löw, S. Gupta, T. Pfau, A. Görlitz, T. 
L. Gustavson, D. E. Pritchard, and W. Ketterle, condmat/0006455). Only two electronic levels are involved in this experiment, but different motional components were coupled by frequency resolved Raman fields, effectively creating a three level type structure typical for EIT.

[3] S. E. Harris, J. E. Field, and A. Imamoğlu, Phys. Rev. Lett. 64, 1107 (1990); M. O. Scully, Phys. Rep. 219, 191 (1992); S. E. Harris, Phys. Today 50, 36 (1997), and references therin.

[4] M. M. Kash, V. A. Sautenkov, A. S. Zibrov, L. Hollberg, G. R. Welch, M. D. Lukin, Y. Rostovtsev, E. S. Fry, and M. O. Scully, Phys. Rev. Lett. 82, 5229 (1999).

[5] A. V. Turukhin, J. A. Musser, V. S. Sudarshanam, M. S. Shahriar, and P.R. Hemmer, quant-ph/0010009).

[6] O. Kocharovskaya, Y. Rostovtsev, and M. O. Scully, (quant-ph/0001058).

[7] S. E. Harris and L. V. Hau, Phys. Rev. Lett. 82, 4611 (1999); M. D. Lukin et al., ibid. 82, 1847 (1999); S. Brandt et al., Phys. Rev. A 56, R1063 (1997); H. Schmidt and A. Imamoğlu, Opt. Lett. 21, 1936 (1996).

[8] M. D. Lukin and A. Imamoglu, Phys. Rev. Lett. 84, 1419 (2000).

[9] M. D. Lukin, S. F. Yelin, and M. Fleischhauer, Phys. Rev. Lett. 84, 4232 (2000).

[10] M. Fleischhauer and M. D. Lukin, Phys. Rev. Lett. 84, 5094 (2000).

[11] A. B. Matsko, Y. V. Rostovtsev, H. Z. Cummins, and M. O. Scully, Phys. Rev. Lett. 84, 5752 (2000).

[12] M. Fleischhauer, M. D. Lukin, A. B. Matsko, and M. O. Scully, Phys. Rev. Lett. 84, 3558 (2000).

[13] G. Morigi and G. Agarwal, Phys. Rev. A 62, 013801, 2000.

[14] L. You, M. Lewenstein, R. J. Glauber, and J. Cooper, Phys. Rev. A 53, 329 (1996).

[15] K. V. Krutitsky, F. Burgbacher, and J. Audretsch, Phys. Rev. A 59, 1517 (1999).

[16] L. Allen and J. H. Eberly, Optical Resonance and Two Level Atoms, (John Wiley, New York, 1975), §7.4, pp. 162-164.

[17] J. Jackson, Classical Electrodynamics, 2nd ed. (Wiley, New York, 1975).

[18] C. M. Bowden and J. P. Dowling, Phys. Rev. A 47, 1247 (1993).

[19] H. Wallis, Phys. Rev. A 56, 2060 (1997).

[20] O. Morice, Y. Castin, and J. Dalibard, Phys. Rev. A 51, 3896 (1995).

[21] J. Ruostekoski and J. Javanainen, Phys. Rev. A 55, 513 (1997).

[22] J. Ruostekoski and J. Javanainen, Phys. Rev. A 56, 2056 (1997).

[23] M. Fleischhauer and S. F. Yelin, Phys. Rev. A 59, 2427 (1999).

[24] K. V. Krutitsky, F. Burgbacher, and J. Audretsch, Laser Phys. 10, 15 (2000).

[25] J. Ruostekoski and J. Javanainen, Phys. Rev. Lett. 82, 4741 (1999).

[26] J. Ruostekoski, Phys. Rev. A 61, 033605 (2000).

[27] D. A. Butts and D. S. Rokhsar, Phys. Rev. A 55, 4346 (1997).

[28] M. Naraschewski and D. M. Stamper-Kurn, Phys. Rev. 
A 58, 2423 (1998).

[29] V. Bagnato, D. E. Pritchard, and D. Kleppner, Phys. Rev. A 35, 4354 (1987).

[30] S. Giorgini, L. P. Pitaevskii, and S. Stringari, J. Low Temp. Phys. 109, 309 (1997).

[31] B. DeMarco and D. S. Jin, Science 285, 1703 (1999).

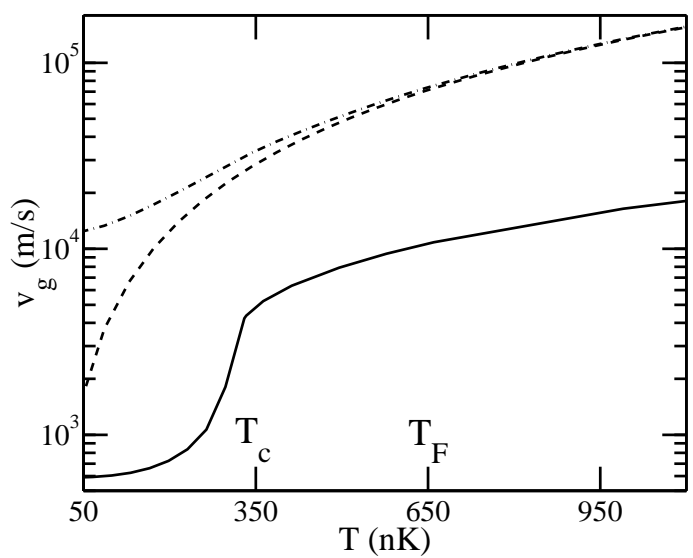

FIG. 1. Temperature dependent group velocity in Fermi (dash-dotted line), Bose (solid line), and Boltzman (dashed line) for two level trapped quantum gases. We used $\mathrm{Na}$ as an example with the following parameters: $N=3.8 \times 10^{6}$, $R=7.5(\mu \mathrm{m}), \omega_{r}=(2 \pi) 69(\mathrm{~Hz}), \epsilon=1 / 3, \gamma=(2 \pi) 10.03$ $(\mathrm{Hz}), a_{\mathrm{sc}}=2.75(\mathrm{~nm}), \omega_{0}=(2 \pi) 5.1 \times 10^{14}(\mathrm{~Hz})$ as in Ref. [1]. For these parameters $R_{B}=17.76(\mu \mathrm{m}), R_{F}=50.04$ $(\mu \mathrm{m}), a_{r}=2.52(\mu \mathrm{m})$, and $a_{\mathrm{ho}}=3.03(\mu \mathrm{m})$. We chose the detuning $\Delta=10 \gamma$ to assure sufficiently transmission.

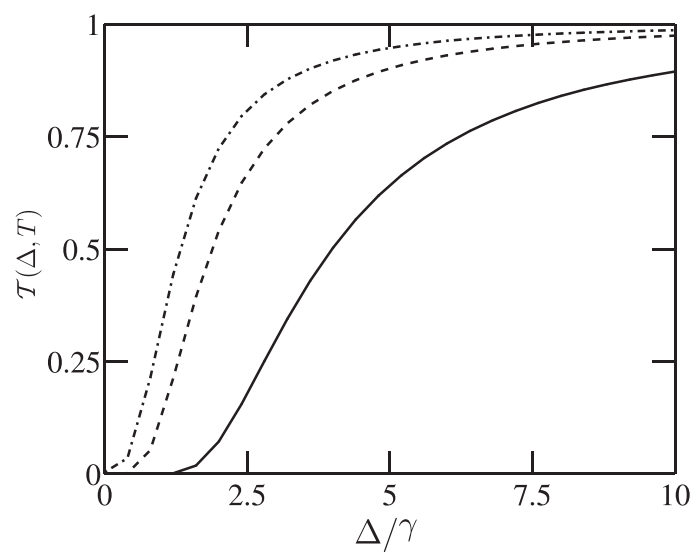

FIG. 2. The same as in Fig. 1, except for the detuning dependence of the transmission coefficient. All three quantum gases are compared for the same temperature $T=T_{c} / 2 \approx T_{F} / 4$. 